\title{
Creación de 'espacios seguros' para niñas adolescentes
}

Wendy Baldwin

Population Council

Follow this and additional works at: https://knowledgecommons.popcouncil.org/departments_sbsr-pgy

Part of the Demography, Population, and Ecology Commons, Family, Life Course, and Society Commons, Gender and Sexuality Commons, International Public Health Commons, and the Medicine and Health Commons How does access to this work benefit you? Let us know!

\section{Recommended Citation}

Baldwin, Wendy. 2011. "Creación de 'espacios seguros' para niñas adolescentes," Promoción de transiciones a la vida adulta sanas, seguras y productivas Resumen no. 39. New York: Population Council. 


\section{Creación de "espacios seguros" para niñas adolescentes}

\section{Elaborado por Wendy Baldwin}

L a adolescencia es una época de muchas transiciones y, a su vez, es el momento en el que se adquieren las habilidades para la vida adulta. Aunque estas habilidades se pueden seguir adquiriendo durante la edad adulta, la adolescencia es el momento en el que se debe crear una base en la educación, habilidades financieras, comportamientos de salud positivos y pensamientos críticos. Para muchas niñas del mundo desarrollado, la oportunidad de moverse libremente en la comunidad se vuelve más limitada en el comienzo de la pubertad. Esta es una medida protectora bien intencionada, pero provoca la limitación de las oportunidades de las niñas de fortalecer las relaciones sociales, ganar las habilidades necesarias y aprender a ser miembros completos de su comunidad.

El Council ha desarrollado un número de programas de "espacios seguros" que abordan las necesidades de las adolescentes vulnerables, especialmente a las niñas. El trabajo comienza con identificar a las niñas que son especialmente vulnerables y después encontrar un espacio físico seguro donde puedan reunirse en grupo. Un mentor entrega un plan de estudios y actúa como un modelo de rol para las niñas. Estos programas varían según la contribución local, pero todos están creados con los elementos clave de un espacio físico seguro, un mentor y una red social o de amistades para las niñas.

\section{¿Qué es un "espacio seguro"?}

Un "espacio seguro" normalmente significa un espacio sólo para niñas.

Esto es un componente importante ya que los espacios públicos normalmente están llenos de hombres. Algunos ejemplos de lugares que se pueden usar son: salones comunitarios, espacios de programas dedicados, escuelas, centros juveniles e incluso contenedores de barcos vacíos. Encontrar un espacio puede involucrar que las niñas ayuden a localizar los lugares donde se sientan seguras y a obtener el permiso para poder usar ese lugar. Por ejemplo, en Egipto, por ley, se dispone de un centro juvenil en cada ciudad para todos los miembros de la comunidad. Sin embargo, desde entonces los centros se usan exclusivamente por niños y hombres, por lo que no se consideran "seguros" para las niñas. Trabajando con la comunidad y los centros juveniles, conseguimos que nos concediesen algún tiempo en los centros, al que se denominó el momento "sólo para niñas", ideal para el programa Ishraq.

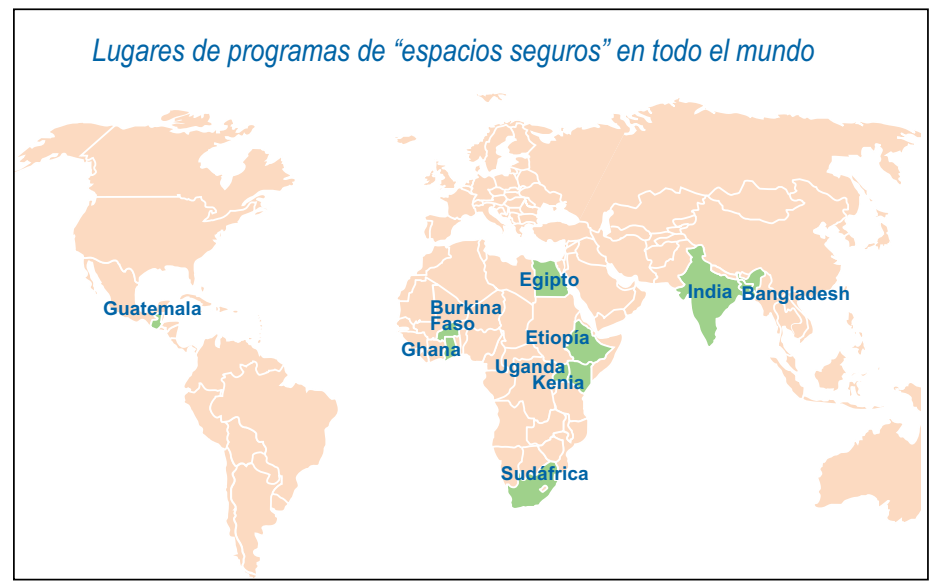

¿Por qué las redes sociales y de amistades son importantes? En muchos entornos la llegada de la pubertad disminuye el acceso de las niñas a las amistades y a la libertad para moverse en la comunidad. La llegada de la pubertad coincide con el abandono escolar, el aumento de las tareas domésticas o la preparación para el matrimonio. Los padres tienen miedo de la seguridad de las niñas o del honor de la familia ya que la madurez sexual significa que las niñas son objeto de los avances de los hombres. Sin embargo, restringir la movilidad lleva a una reducción del contacto con sus amistades. Muchos estudios apuntan a la importancia de las redes sociales para tener una buena salud (Berkman y Syme 1979; Marmot et al. 1991; Kawachi et al. 1996) y para conseguir recursos prácticos. Las niñas adolescentes tienen menos probabilidades que los niños de tener amistades más duraderas, por ejemplo si van a algún sitio necesitarán un lugar donde poder estar, un amigo al que pedir dinero prestado en caso de necesidad o recursos que puedan protegerlas si están en peligro en casa (Erulkar et. al. 2004; Hallman 2009). Los programas de espacios seguros hacen que exista un contacto social entre las niñas para ayudar a promover dichas redes sociales.

\section{¿Cuál es el rol de los mentores?}

En general, los mentores son mujeres jóvenes de la comunidad con las que las niñas se pueden identificar. Además de implementar el plan 


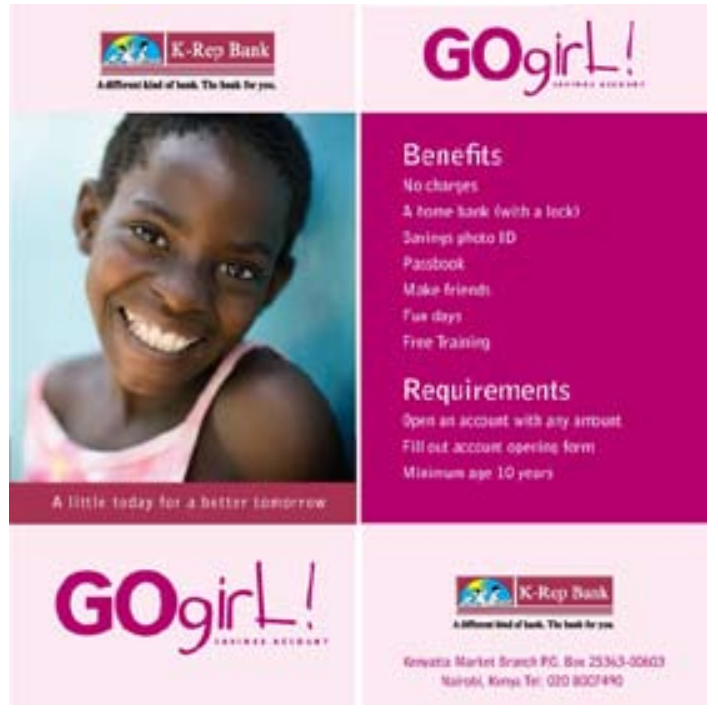

de estudios, los mentores sirven como modelos de rol para las niñas. Al contrario que los programas paritarios, los mentores son un poco más mayores que las niñas del programa. Incluso en entornos con dificultades, frecuentemente hay niñas que se han ido antes de la escuela que otras $y$ tienen las habilidades necesarias para servir como mentores. En otros entornos las niñas pueden afrontar las necesidades específicas que se le piden a las mujeres mayores para ser mentores. Por ejemplo, en Addis Ababa, Etiopía, las niñas que participan en el programa de espacios seguros son también empleadas domésticas. Estas niñas necesitan ayuda para negociar con sus empleadores y para apelar a sus derechos legales, por lo que los mentores adultos son más efectivos en este entorno.

Creamos un modelo de liderazgo en cascada mediante estos programas para cultivar futuros líderes y ayudar a asegurar la sostenibilidad. En la mayoría de los programas somos capaces de identificar a las niñas que están más interesadas en desarrollar sus habilidades, en tener más educación y en demostrar su potencial de líderes. Cuando están entrenadas para liderar y hacer de mentores de los nuevos grupos, se les proporciona una actividad paga, lo que permite que el programa se extienda a la comunidad.

\section{¿Quienes son las niñas?}

Las niñas son de diferentes edades y el contenido de los programas es diferente según el lugar y las necesidades de las mismas. Aunque la mayoría de los programas inicialmente tuvieron como objetivo adolescentes de entre 15 y 17 años, hay lugares que tuvieron que aumentar cada vez más a edades más tempranas, de entre 10 y 14 años, ya que se debe conseguir llegar a ellas antes de la edad de abandono escolar o de los matrimonios prematuros.

Los programas varían según la frecuencia con la que se juntan los grupos y la extensión del programa. La mayoria de los programas se reúne una o dos veces a la semana, mientras que otros se reúnen cuatro veces a la semana durante tres horas al día. El más intenso es el de Ishraq, donde la mayoría de las niñas no va a la escuela y se necesita de tiempo extra para alcanzar los objetivos de alfabetización y de cálculo del programa. La mayoría de los programas dura entre un año y dos años y medio. La intensidad de la intervención está unida a las necesidades de las niñas y a la habilidad de encajar el programa con sus obligaciones como la escuela, las tareas domésticas o el trabajo del campo.

La mayoría de los programas incluyen capacitación para la alfabetización, aunque este aspecto es más importante en algunos entornos que en otros. Todos los programas incluyen educación sobre el comportamiento de la salud, especialmente con lo relacionado a la salud reproductiva y sexual y están ligados a los servicios de la salud de la comunidad. En todas las zonas, se incluye el tema de la violencia de género ya que afecta a muchas niñas y mujeres jóvenes. En las zonas donde hay más matrimonios prematuros, uno de los mayores focos del programa es la postergación del matrimonio. Otro objetivo es ayudar a las niñas a desarrollar las aptitudes de la vida y el conocimiento como una negociación interpersonal, la experiencia para pensar en los objetivos de la vida y los elementos de la ciudadanía. Las capacidades financieras, históricamente excluidas de la mayoría de los planes de estudio sobre aptitudes de la vida, varían desde la alfabetización financiera básica hasta la educación de las capacidades financieras más amplias y los programas de ahorro. Los programas no tienen planes de estudio rígidos sino que son flexibles, para asegurar que se traten los temas pero también que se les de tiempo a las niñas para que puedan plantear los temas que las preocupan.

\section{Lecciones aprendidas}

Los datos existentes pueden usarse para identificar a las niñas que son vulnerables y que fueron excluidas de otros programas juveniles. Por ejemplo, las Encuestas Demográficas y de Salud (Demographic and Health Surveys) nos permiten identificar las zonas donde las niñas no van a la escuela, no viven con los padres o están en riesgo de contraer matrimonio prematuro. Después de identificar las zonas de necesidad, es posible trabajar con los padres y con los líderes de la comunidad para establecer un programa, al que muchas comunidades dan la bienvenida. También, hay un interés claro por parte de las niñas: no es insólito que tengan más interés de lo esperado, por lo que es necesario organizar listas de espera para los programas. Sin embargo, las demandas de otras obligaciones pueden ser un problema de asistencia. Desde el principio, los programas intentan buscar tiempo que se corresponda con los tiempos en que estén disponibles las niñas, una razón frecuente de no asistir a las sesiones es que las niñas tienen que realizar trabajos domésticos o cuidar de algún hermano.

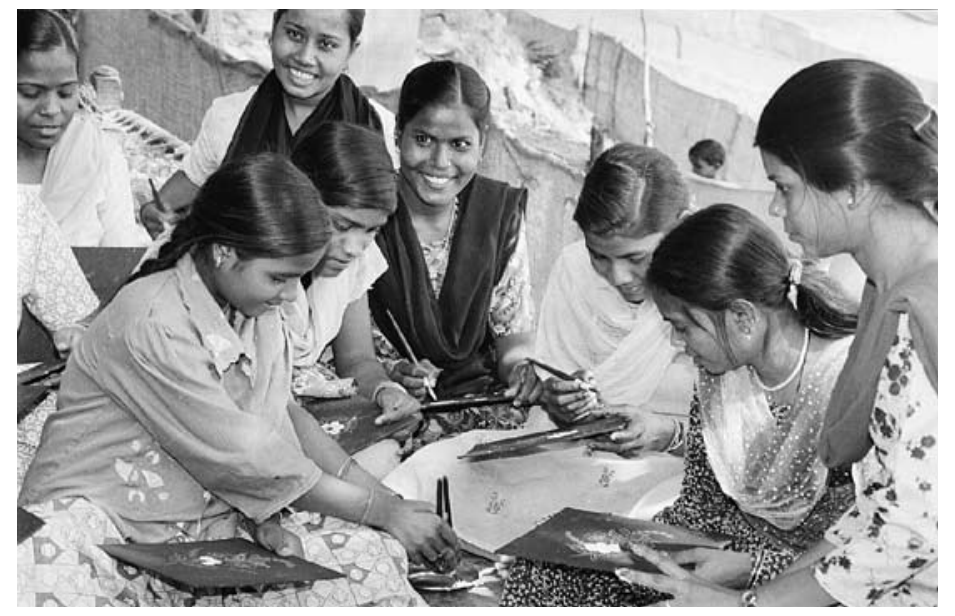

2 - Visite www.popcouncil.org/publications/serialsbriefs/TABriefs.asp para obtener todos los resúmenes de la serie Promoting healthy, safe, and productive transitions to adulthood (Promoción de transiciones a la vida adulta sanas, seguras y productivas). 
Evaluar el impacto de estos programas no es sencillo porque muchos efectos no se pueden observar hasta que pasan los años. Algunos elementos de los programas son sencillos de evaluar y los resultados son prometedores. Por ejemplo, las niñas matriculadas en el proyecto egipcio tenían poco o ningún estudio. Entre las niñas que permanecieron durante 30 meses en el programa y se presentaron al examen, el 92 por ciento superó la prueba de alfabetización. Otros estudios muestran mejoras evidentes en el conocimiento de los problemas de la salud y amplían los objetivos de vida (Hallman 2008; Erulkar et al. 2011). Aunque es más fácil medir el impacto de un programa en uno o dos dominios específicos, estos avances tienen un impacto en toda la trayectoria de la vida de una niña.

\section{Postergación del matrimonio infantil y apoyo a las madres jóvenes}

- Padres primerizos ("First Time Parents"), India - Un grupo especial con la necesidad de programas de espacios seguros son los padres primerizos, especialmente en zonas donde la edad de matrimonio es muy baja. Trabajando en centros comunitarios, el programa alcanzó a tener 1.700 niñas en dos regiones, West Bengal y Gujarat.

- Madres educadoras ("Mères Éducatrices"), Burkina Faso - 4.000 niñas de entre 10 y 19 años se matricularon en los programas en los centros escolares y sanitarios de sus pueblos. El objetivo de este programa fue postergar los matrimonios infantiles y capacitar a las madres jóvenes como educadoras para apoyar a otras madres jóvenes en sus casas.

- Berhane Hewan, Etiopía - "Luz durante la Víspera" ("Light for Eve") alcanzó la cantidad de 12.000 niñas casadas y no casadas de entre 10 y 19 años en una región rural de Etiopía donde la mitad de ellas están casadas desde los 15 años. Las estructuras kebele de los pueblos proporcionan los lugares y con el apoyo del gobierno el programa alcanzará 72.000 niñas.

\section{Desarrollo de la alfabetización financiera}

- Siyakha Nentsha "Construir con gente joven" ("Building with Young People"), Sudáfrica - Este programa, que tiene ventaja por la amplia cantidad de matriculaciones en la escuela en KwaZulu Natal, trabaja con 1.100 niñas y niños en sus propias escuelas. Un componente es la alfabetización financiera y los planes de estudio fueron certificados por el gobierno de modo que completando esta parte del programa se proporciona una credencial comercial para la juventud.

- Ahorros seguros e inteligentes ("Safe and Smart Savings"), Kenia Este programa de ahorro es una extensión de uno de los programas de espacios seguros más reciente, Binti Pamoja. Localizado en el barrio de Kibera, el programa va más allá de la educación financiera a un programa bancario en relación con dos bancos de Nairobi. El programa se está extendiendo ahora a Uganda. Actualmente cuenta con 2.300 jóvenes, pero cuando la fase esté totalmente extendida contará con alrededor de 20.000 participantes.

- Kishori Abhijan, Bangladesh ó Trabajando con clubes de adolescentes en zonas rurales y en escuelas técnicas no formales de las zonas urbanas, el programa cuenta con niñas de entre 15 y 19 años para promover la educación financiera mediante relaciones con los mentores. Esto es una prueba estructurada del valor de añadir mentores a las aproximaciones educativas financieras tradicionales.

\section{Ampliación de las oportunidades de la vida}

- Ishraq "Amanecer" ("Sunrise”), Egipto - En el Alto Egipto rural, el programa Ishraq ha trabajado con 1.800 niñas de entre 12 y 15 años para animarlas a volver a entrar en la escolarización formal e incrementar la educación de la salud y la alfabetización. Ganando el apoyo de las comunidades fuertes y del gobierno, el programa se está expandiendo y se espera que se convierta en un patrocinador del gobierno en el futuro próximo.

- Abriendo Oportunidades, Guatemala - El programa cuenta con 1.260 niñas de Maya en dos grupos según la edad, de entre 8 y 12 años y de 13 y 18 años. Los programas se realizan en los salones comunitarios $y$ en las escuelas y utilizan un modelo de "mentor en cascada" de modo que las niñas podrán avanzar en posiciones de liderazgo en el programa.

- Biruh Tesfa "Futuro brillante" ("Bright Future"), Etiopía - Comenzando en las zonas de barrio urbanas como las de Addis Ababa, niñas de entre 10 y 19 años de Etiopía (incluyendo a las trabajadoras domésticas) formaron parte del Biruh Tesfa. Trabajando en salones comunitarios donados por los kebele, o por el gobierno local, el programa cuenta con 16.500 niñas y crecerá hasta 30.000 con la suma de 12 ciudades más.

- "Niñas Inteligentes" ("Smart Girls"), Ghana - El programa más reciente es el programa "Niñas Inteligentes" de Tema, Ghana. Basado en las experiencias de otros programas urbanos, "Niñas Inteligentes" cuenta con 89 niñas en el programa piloto. Niñas de entre 12 y 15 años ayudaron a renovar un edificio escolar vacante que será el espacio donde tendrá lugar el programa.

\section{Direcciones futuras}

Como los programas de "espacios seguros" se mueven más allá de una fase piloto, hay una oportunidad de aprender más sobre el beneficio relativo de los componentes del programa primario. Por ejemplo, Berhane Hewan está probando ahora la contribución relativa de las conversaciones de la comunidad, la evaluación de las niñas y los componentes que incentivan a postergar la edad de matrimonio. Estas evaluaciones son importantes

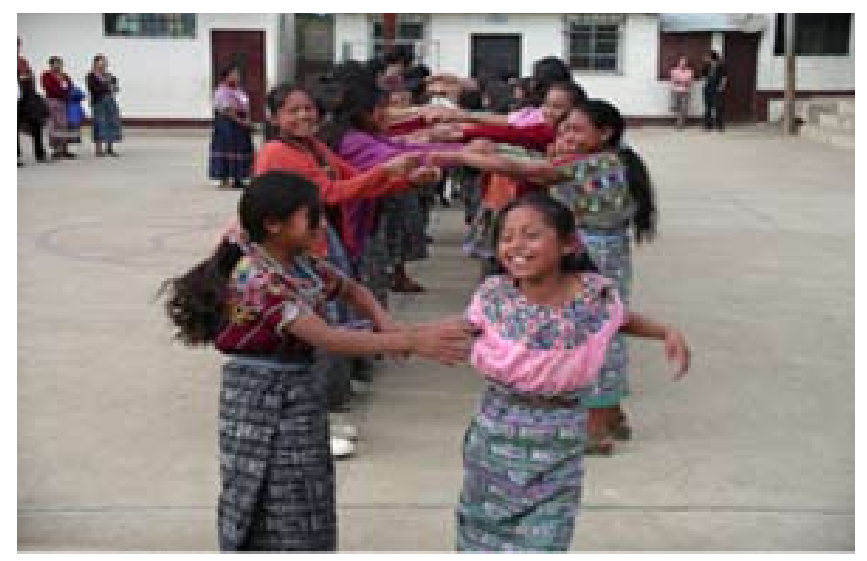


por lo que buscamos programas más eficientes para ampliar mientras nos aseguramos de que se mantengan los componentes importantes. También, como programas desarrollados, se puede examinar el impacto de los programas en la vida de las niñas, más allá del tiempo que pierden en la intervención. ¿Las niñas postergan el matrimonio y la maternidad? ¿Son más activas económicamente? ¿Su nuevo sentido de un lugar en la comunidad resulta en un compromiso cívico mayor? Ya que las niñas de los programas se casarán en la mayoría de los casos con niños de su comunidad, ¿hay intervenciones clave como alternativas a la violencia para resolver discusiones, ayudar con los presupuestos y los ahorros 0 comunicar mensajes de salud reproductiva y sexual básicos a los niños? Algunas direcciones futuras quizás impliquen pensar sobre como integrar el aprendizaje de los programas de espacios seguros en un ambiente cambiante para las niñas. Por ejemplo, con el incremento de las responsabilidades de la escuela, ¿la mayoría de las niñas intenta asistir ahora a la escuela? Si las niñas ya están en la escuela, ¿deberíamos alterar alguno de los contenidos del programa o intentar buscar que se integre algún contenido (por ejemplo, educación sobre la sexualidad) en los programas escolares? ¿Si los bancos móviles se extienden más, proporcionarán nuevas oportunidades para los programas de ahorro de las niñas? Como buscamos mejorar las oportunidades de las niñas, necesitamos evaluar constantemente el modo en el cual su entorno dinámico está cambiando y su efecto en cómo hacemos nuestro trabajo.

\section{Referencias y publicaciones relacionadas}

Amin, Sajeda. 2011. "Empowering adolescent girls in rural Bangladesh: Kishori Abhijan," Promoting Healthy, Safe, and Productive Transitions to Adulthood Brief no. 13. Nueva York: Population Council.

Austrian, Karen. 2011. "Safe spaces, financial education, and savings for adolescent girls in Kenya," Promoting Healthy, Safe, and Productive Transitions to Adulthood Brief no. 29. Nueva York: Population Council.

- 2010. Girl-Centered Program Design: A Toolkit to Develop, Strengthen \& Expand Adolescent Girls Programs. Nairobi: Population Council.

Berkman, Lisa and S. Leonard Syme. 1979. "Social networks, host resistance, and mortality: A nine-year follow-up study of Alameda county residents," American Journal of Epidemiology 109:186-204.

Brady, Martha. 2003. "Chapter 7-Safe spaces for adolescent girls," en Adolescent and Youth Sexual and Reproductive Health: Charting Directions for a Second Generation of Programming -Background Document for the Meeting. Nueva York: UNFPA, pág.155-176.

Catino, Jennifer, Alejandra Colom, and Marta Julia Ruiz. 2011. "Equipping Mayan girls to improve their lives," Promoting Healthy, Safe, and Productive Transitions to Adulthood Brief no. 5. Nueva York: Population Council.
Engebresten, Sarah. 2011. "Piloting a safe spaces, asset-building program for adolescent girls in urban Ghana," Promoting Healthy, Safe, and Productive Transitions to Adulthood Brief no. 34. Nueva York: Population Council.

Engebresten, Sarah, Gisele Kabore, Martha Brady and Lydia Saloucou. 2011. "Addressing the needs of girls at-risk of early marriage and married adolescent girls in Burkina Faso," Promoting Healthy, Safe, and Productive Transitions to Adulthood Brief no. 9. Nueva York: Population Council.

Erulkar, Annabel S., Tekle-Ab Mekbib, Negussie Simie and Tsehai Gulema. 2004. "Adolescent life in low income and slum areas of Addis Ababa, Ethiopia." Nueva York: Population Council

Erulkar, Annabel, Belaynesh Semunegus and Gebeyehu Mekonnen. 2011. "Biruh Tesfa ('Bright Future') Program Provides Domestic Workers, Orphans \& Migrants in Urban Ethiopia with Social Support, HIV Education \& Skills," Promoting Healthy, Safe, and Productive Transitions to Adulthood Brief no. 21. Nueva York: Population Council.

Hallman, Kelly. 2008. "Researching the determinants of vulnerability to HIV among adolescents." IDS Bulletin 39: 36 -44.

_ 2009. "Social exclusion: The gendering of adolescent HIV risk in South Africa" en J. Klot y V. Nguyen (eds.), The Fourth Wave: Violence, Gender, Culture, and HIV in the $21^{\text {st }}$ Century. Social Science Research Council y UNESCO. <http://blogs.ssrc.org/fourthwave/>. Acceso el 5 de mayo de 2011.

Hallman, Kelly, Kasthuri Govender, Eva Roca, Rob Pattman, Emmanual Mbatha and Deevia Bhana. 2011. "Enhancing financial literacy, HIVIAIDS skills, and safe social spaces among vulnerable South African youth," Promoting Healthy, Safe, and Productive Transitions to Adulthood Brief no. 4. Nueva York: Population Council.

Kawachi, I., G.A. Colditz, A. Ascherio, et al. 1996. "A prospective study of social networks in relation to total mortality and cardiovascular disease in men in the US," Journal of Epidemiology and Community Health 50: 245 -251.

Kayanwala, Shveta. 2011. "Influencing girlsí lives: Acceptability and effectiveness of a livelihoods skill building intervention in Gujarat," Promoting Healthy, Safe, and Productive Transitions to Adulthood Brief no. 18. Nueva York: Population Council.

Marmot, Michal G., G.D. Smith, S. Stansfeld, et al. 1991. "Health inequalities among British civil servants: The Whitehall II study," Lancet 337:1387-1393.

Muthengi, Eunice y Annabel Erulkar. 2011. "Delaying early marriage among disadvantaged rural girls in Amhara, Ethiopia through social support, education and community awareness," Promoting Healthy, Safe, and Productive Transitions to Adulthood Brief no. 20. Nueva York: Population Council.

Santhya, K.G., Nicole Haberland, Arup Das, et al. 2008. Empowering Married Young Women and Improving their Sexual and Reproductive Health: Effects of the First-time Parents Project. Nueva Delhi: Population Council.

Zibanai, Nadia and Martha Brady. 2011. "Scaling up asset-building programs for marginalized adolescent girls in socially conservative settings: The Ishraq program in rural Upper Egypt," Promoting Healthy, Safe, and Productive Transitions to Adulthood Brief no. 12. Nueva York: Population Council.

\section{Q Population Council}

El Population Council cambia la manera en la que el mundo piensa acerca de los problemas de salud y desarrollo. Buscamos entender las causas y las consecuencias de la desigualdad de género y las disparidades en las oportunidades que surgen durante la adolescencia. Brindamos las evidencias para desarrollar mejores programas y políticas prácticas que garanticen una transición exitosa y productiva hacia la edad adulta en los países en vías de desarrollo. www.popcouncil.org

(c) 2011 The Population Council, Inc.

4 - Visite www.popcouncil.org/publications/serialsbriefs/TABriefs.asp para obtener todos los resúmenes de la serie Promoting healthy, safe, and productive transitions to adulthood (Promoción de transiciones a la vida adulta sanas, seguras y productivas). 